\title{
Jenseits von Nachfrage und Angebot
}

Innovationspolitik ist zumeist Industriepolitik. Dabei wird die hohe gesellschaftliche Bedeutung von unspektakulären, nicht kommerziellen Alltagsinnovationen unterschätzt. Innovationspolitik muss daher auch diese kollaborativ geschaffenen Innovationen in den Blick nehmen.

Von Philine Warnke

D as Konzept des Innovationssystems erhebt den Anspruch, „alle wichtigen ökonomischen, sozialen, politischen, organisatorischen, institutionellen und andere Faktoren, die die Entwicklung, Diffusion und Nutzung von Innovationen beeinflussen“ (Edquist 2005, S. 108, eigene Übersetzung), zu erfassen, um auf dieser Basis innovationspolitische Maßnahmen entwickeln zu können.

Daher liegt es nahe, die Erkenntnisse verschiedener aktueller Forschungsstränge aufzugreifen, die auf den maßgeblichen Beitrag vielfältiger „Bottom-up“-Akteure zum Innovationsgeschehen hinweisen.

\section{Wechselspiel gesellschaftlicher Prozesse}

Studien der sozialwissenschaftlichen Technikforschung zeigen schon seit Langem, dass Innovationsprozesse durch ein komplexes Wechselspiel gesellschaftlicher Prozesse mit wechselnden Rollen der beteiligten Akteure gekennzeichnet sind (Bijker/Law 1997). Insbesondere wurde die zentrale Rolle von Nutzergruppen (Pinch/Kline 1996) und gesellschaftlichen Erwartungen (Konrad 2004) herausgestellt.

Auch Innovationsstudien haben die aktive Rolle von Nutzerinnen und Nutzern bei der Generierung von Innovation hervorgehoben (Reichwald/Piller 2006; von Hippel 2006). Beispiele aus einer Reihe von Sektoren zeigen, dass viele Nutzer allein, in Communities oder zusammen mit Unternehmen erhebliche Beiträge zum Innovationsprozess leisten. Noch grundlegender argumentiert Peter Swann (2014).

\section{Alltagsinnovationen}

Er hebt die hohe gesellschaftliche Bedeutung von unspektakulären, nicht kommerziellen Alltagsinnovationen (Common Innovations), die tagtäglich von Graswurzelakteuren geschaf- fen werden, hervor. Zunehmende Aufmerksamkeit wird unter dem Begriff soziale Innovation auch gesellschaftlichen Akteuren zuteil, die neue soziale Praktiken kreieren, um Lösungen für eigene oder Community-Bedarfe zu erzielen (Howaldt/ Schwarz 2010).

Schließlich verweist ein weiterer Forschungsstrang auf die wachsende Bedeutung nicht marktvermittelter, selbst organisierter Lösungsentwicklung durch Gruppen von Individuen mit unterschiedlichen Motivationen und Kompetenzen (Benkler 2013; Raasch et al. 2008; Wittke/Hanekop 2011).

\section{Kooperation und Experimentieren als Innovationstreiber}

Treiber sind die durch das Internet massiv gesunkenen Kosten von Kooperation. Insbesondere im Softwarebereich sind maßgebliche Innovationen mit hohem ökonomischen Wert auf diese Weise zustande gekommen so etwa das GNU/LinuxBetriebssystem und der Apache-Webserver, die von vielen etablierten Firmen wie IBM genutzt werden. Das kollaborativ entwickelte Internetlexikon Wikipedia ist eine der am meisten genutzten Webseiten der Welt.

Kollaborative Innovationsformen ermöglichen ständiges iteratives Experimentieren. Damit sind sie flexibler und vielfältig anpassbar, was besonders in Situationen hoher Unsicherheit, wie sie im Falle komplexer gesellschaftlicher Herausforderungen wie der Nachhaltigkeit vorliegen, vorteilhaft ist. Eine Reihe von Autoren fordert angesichts dieser Entwicklung eine Neuausrichtung der Innovationsforschung und verstärkte Forschungsanstrengungen zu kooperativen Innovationsformen (Baldwin/von Hippel 2010; Bonaccorsi/Rossi 2003; Wittke/Hanekop 2011).

\section{Schlussfolgerung}

Die geschilderten Forschungsstränge zeigen, dass Bottomup-Akteure maßgebliche Beiträge zur „Angebotsseite“ von Innovation leisten. Das Verständnis des Innovationssystems muss demnach deutlich erweitert werden, wenn der Anspruch aufrechterhalten werden soll, die für Innovation relevanten Akteure und Institutionen zu erfassen. Dabei werden nicht nur neue Elemente, sondern auch neue Strukturen hinzukommen müssen (Warnke et al. 2015).

Diese erweiterte Perspektive auf die Landschaft der Innovationsakteure sollte sich auch in innovationspolitischen Ansätzen niederschlagen. Ebenso wie die klassischen Akteure 


\section{„Das Verständnis des Innovations- systems muss deutlich erweitert werden, denn Bottom-up-Akteure leisten maßgebliche Beiträge zur ,Angebotsseite“ von Innovation."}

in Universitäten und Firmen, benötigen auch die Bottom-upAkteure Plattformen und Infrastrukturen wie etwa die OpenSource-Lizenzen.

\section{Bottom-up-Akteure in den Fokus von Innovationspolitik}

Einige Foresight-Prozesse wie „Innovation Futures INFU“ (Schirrmeister/Warnke 2013) und der B MB F-Foresight-Prozess (Zweck et al. 2015) haben ausgemalt, wie Infrastrukturen der Zukunft aussehen könnten, die ein „Kollektives Experimentieren“ (Joly et al. 2010) über die gesamte Vielfalt der Akteurspalette ermöglichen [1].

Solche Ansätze weisen über die Trennung zwischen angebots- und bedarfsseitigen Instrumenten hinaus und fokussieren stattdessen auf deren zunehmend hybride Schnittstelle. Insbesondere die „missionsorientierte Innovationspolitik“, die den Anspruch erhebt, auch gesellschaftliche Herausforderungen zu adressieren (Daimer et al. 2012; Foray et al. 2012), dürfte von einer solchen Erweiterung der Perspektive profitieren.

\section{Anmerkungen}

[1] Weitere Informationen zum BMBF-Foresight-Prozess im Internet unter: www.bmbf.de/de/mit-foresight-in-die-zukunft-schauen-930.html

\section{Literatur}

Baldwin, C./Hippel, E. von (2010): Modeling a Paradigm Shift: From Producer Innovation to User and Open Collaborative Innovation. MIT Sloan School of Management Working Paper No. 4764-09/Harvard Business School Finance Working Paper No. 10-038.

Benkler, Y. (2013): Peer production and cooperation. In: Bauer, J. M./Latzer, M. (Hrsg.): Handbook on the Economics of the Internet. Northhampton, Edward Elgar.

Bijker, W. E./Law, J. (1997): Shaping technology/building society. Studies in sociotechnical change, Inside technology. Cambridge/Mass., MIT Press.

Bonaccorsi, A./Rossi, C. (2003): Why Open Source software can succeed. In: Research Policy 32/7. S. 1243-1258.

Daimer, S./Hufnagel, M./Warnke, P. (2012): Challenge-oriented policy-making and innovation systems theory: reconsidering systemic instruments. In: Innovation system revisited - Experiences from 40 years of Fraunhofer ISI research. Stuttgart, Fraunhofer Verlag. S. 217-234.

Edquist, C. (2005): Systems of Innovation: Perspectives and Challenges. In: Fagerberg, J./Mowery, D. C./Nelson, R. R. (Hrsg.): The Oxford Handboook of Innovation. New York, Oxford Univ. Press. S. 181-208.
Foray, D./Mowery, D. C./Nelson, R. R. (2012): Public R\&D and social challenges: What lessons from mission R\&D programs? In: Research Policy 41/10. S. 1697-1702.

Howaldt, J./Schwarz, M. (2010): Social Innovation: Concepts, Research Fields and International Trends. In: Henning, K./Hees, F. (Hrsg.): Studies for Innovation in a Modern Working Environment - International Monitoring. Volume 5. Aachen.

Joly, P.-B./Rip, A./Callon, M. (2010): Re-Inventing Innovation. In: Arentsen, M. J./Van Rossum, W./Steenge, A. E. (Hrsg.): Governance of Innovation: Firms, Clusters and Institutions in a Changing Setting. Cheltenham, Edward Elgar. S. 19-32.

Konrad, K. (2004): Prägende Erwartungen: Szenarien als Schrittmacher der Technikentwicklung. Berlin, Edition Sigma.

Pinch, T./Kline, R. (1996): Users as Agents of Technological Change. The Social Construction of the Automobile in Rural America. In: Technology and Culture 37. S. 763-795.

Raasch, C./Herstatt, C./Abdelkafi, N. (2008): Open Source Innovation Characteristics and Applicability Outside the Software Industry. TIM-Working Paper. Hamburg, Technische Universität Hamburg-Harburg.

Reichwald, R./Piller, F. (2006): Interaktive Wertschöpfung: Open Innovation. Individualisierung und neue Formen der Arbeitsteilung 1. Wiesbaden, Gabler.

Schirrmeister, E./Warnke, P. (2013): Envisioning structural transformation. Lessons from a foresight project on the future of innovation. In: Technological Forecasting and Social Change 80/3. S. 453-466.

Swann, P. (2014): Common Innovation: How We Create the Wealth of Nations. Cheltenham, Edward Elgar.

von Hippel, E. (2006): Democratizing Innovation: The evolving phenomenon of User Innovation. In: Kahin, B./Foray, D. (Hrsg.): Advancing Knowledge and the Knowledge Economy. S. 237-255.

Warnke, P./Koschatzky, K./Som, O./Stahlecker, T./Nabitz, L./Braungardt, S./Cuhls, K./Dönitz, E. J./Güth, S./Zenker, A. (2015): Opening up the innovation systems framework towards new actors and institutions. The 2015 Annual Conference of the EU-SPRI Forum. Im Internet unter: euspri-helsinki2015.org/abstracts/pdf/4C1_EU-SPRI_Helsinki_2015_ Innovation_System_Framework_Warnke.pdf

Wittke, V./Hanekop, H. (2011): New Forms of Collaborative Innovation and Production on the Internet. In: Wittke, V./Hanekop, H. (Hrsg.): New Forms of Collaborative Innovation and Production on the Internet. Göttingen, Universitätsverlag Göttingen. S. 9-29.

Zweck, A./Holtmannspötter, D./Braun, M./Erdmann, L./Hirt, M./Kimpeler, S. (2015): Geschichten aus der Zukunft 2030. Ergebnisband 3 zur Suchphase von BMBF-Foresight Zyklus II. Düsseldorf. 